\title{
Persistent photoconductivity induced by electric currents in epitaxial thin films of $\mathrm{Pr}_{0.7} \mathrm{Sr}_{0.3} \mathrm{MnO}_{3}$
}

\author{
J. F. Wang and J. Gao a) \\ Department of Physics, The University of Hong Kong, Pokfulam Road, Hong Kong
}

(Presented 15 November 2010; received 23 September 2010; accepted 22 October 2010; published online 17 March 2011)

The effects of electric currents on the photoconductivity of epitaxial $\mathrm{Pr}_{0.7} \mathrm{Sr}_{0.3} \mathrm{MnO}_{3}$ films were studied. The as-prepared films showed a transient photoconductivity in a wide temperature range. After the films were processed by a current of density $\sim 10^{5} \mathrm{~A} / \mathrm{cm}^{2}$ for certain duration at room temperature, a highly resistive and metastable state was excited at low temperature. This induced state exhibited significant electroresistance and persistent photoconductivity. The phenomena found in films grown on $\mathrm{LaAlO}_{3}$ were similar to those on $\mathrm{SrTiO}_{3}$. The observed effects might be related to the coexistence and instability of the multiphases in manganites. (C) 2011 American Institute of Physics. [doi:10.1063/1.3536458]

\section{INTRODUCTION}

Doped manganites have attracted great interests for their prominent properties and rich physics. ${ }^{1-5}$ Early studies indicated that in such materials strong coupling between the local $t_{2 g}$ spin and mobile $e_{g}$ electron results in simultaneous conductivity and magnetism, known as double exchange interaction (DE). Later electron-lattice interaction, namely Jahn-Teller (JT) distortion, was found to be also significant. Actually, different degrees of freedom, including spin, charge, lattice, and orbit, are interconnected in manganites. These cross-couplings give rise to diverse electronic ground states, which are energetically comparable to each other and constitute a multivalley energy landscape. As a consequence, different electronic states may coexist in a single chemical phase. This characterizes phase separation (PS) and demonstrates the complexity in manganites. On the other hand, it may be favorable for practical applications, because the delicate balances between different phases can be shifted by small external stimuli, such as magnetic field, electric current/field, pressure, and light exposure.

The resistance change upon an electric field/current in a charge-ordered single crystal $\left(\operatorname{Pr}_{0.7} \mathrm{Ca}_{0.3} \mathrm{MnO}_{3}\right)$ was as large as several orders. ${ }^{6}$ In materials with other ground states, the electric field/current-induced effects were also pronounced. For example, in $\mathrm{La}_{0.8} \mathrm{Ca}_{0.2} \mathrm{MnO}_{3}$ films, asymmetric conduction and metastable states, which were readily susceptible to weak currents, could be excited by large currents. ${ }^{7,8}$ Light illumination-induced effects in manganites have also gained considerable attention. ${ }^{9-16}$ Early studies found that the chargeordered (CO) state in $\mathrm{Pr}_{0.7} \mathrm{Ca}_{0.3} \mathrm{MnO}_{3}$ single crystals could be collapsed by light and x ray. ${ }^{9,10}$ Other reported photoinduced phenomena are various, including photoinduced antiferromagnetic-ferromagnetic phase tansition, ${ }^{11}$ photoinduced coherent magnetization precession, ${ }^{12}$ and persistent photoconductivity (PPC) ${ }^{13,14}$ Although the studies on current- and light-induced effects on manganites are quite active, the exact mechanisms

\footnotetext{
a) Author to whom correspondence should be addressed. Electronic mail: jugao@hku.hk.
}

have not been fully understood and there have been scanty explorations on photoresponse of current-induced state. In this paper, we report the current-induced persistent photoconductivity in epitaxial thin films of $\mathrm{Pr}_{0.7} \mathrm{Sr}_{0.3} \mathrm{MnO}_{3}$ (PSMO).

\section{EXPERIMENTAL PROCEDURES}

PSMO films were deposited by pulsed laser deposition (PLD) in $70 \mathrm{~Pa} \mathrm{O}_{2}$ and at $700{ }^{\circ} \mathrm{C}$ onto (001) $\mathrm{SrTiO}_{3}$ (STO) and $\mathrm{LaAlO}_{3}$ (LAO) single crystalline substrates. After deposition, the films were annealed in situ at grown temperature in 1 bar oxygen for half an hour. The thickness of the samples is $\sim 300$ $\mathrm{nm}$, as controlled by deposition time. As suggested by the x-ray diffraction spectra $(\theta-2 \theta$ scan and $\Phi$ scan), the obtained films on both substrates have good crystallinity and epitaxy. To get a homogenous illumination and study the current-induced effects, microbridges [ $\sim 30 \mu \mathrm{m} \times 100 \mu \mathrm{m}$, see Fig. 1(inset)] were made by using conventional photolithography. Ohmic contacts were achieved by evaporating silver electrodes onto the films and a standard four-probe method was used for electric measurements. A semiconductor laser (wavelength $\lambda=532 \mathrm{~nm}$ ) with the power density of $\sim 2 \mathrm{~mW} / \mathrm{mm}^{2}$ was used to illuminate the sample through the optical window of the cryostat.

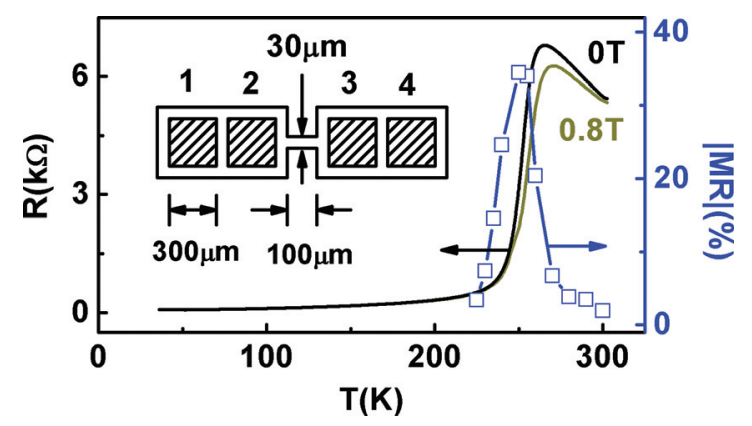

FIG. 1. (Color online) Temperature dependence of resistance $\left(\mu_{0} H=0\right.$ and $0.8 \mathrm{~T})$ and MR ratio for the patterned PSMO film on LAO. MR is defined as $\mathrm{MR}=[R(\mathrm{H})-R(0)] / R(0)$, where $R(H)$ and $R(0)$ are the resistance with and without a magnetic field, respectively. (Inset) Schematic picture of the microbridges. 


\section{RESULTS AND DISCUSSIONS}

Figure 1 depicts the temperature dependence of resistance and magnetoresistance (MR) for a PSMO film on LAO. As a typical feature of doped manganites, the grown film shows a metal-to-insulator transition at $T_{P} \sim 260 \mathrm{~K}$, slightly higher than that of the bulk PSMO ( $245 \mathrm{~K})$. With the application of a magnetic field, the resistance is reduced and $T_{P}$ is shifted to a higher temperature. The maximum MR ratio is about $34 \%$ at $0.8 \mathrm{~T}$.

Figure 2 shows the photoinduced changes of resistance for the as-prepared PSMO films on STO. The photoinduced resistance LR is defined as $[R(L)-R(0)] / R(0)$, where $R(L)$ and $R(0)$ are the resistance with and without light illumination, respectively. Several characteristics can be noted. First, under light illumination, the resistance is increased in the metallic region $(d R / d T>0)$, while that is decreased in insulating region $(d R / d T<0)$. Second, after the light was turned off the resistance recovers to the initial value, indicating a transient photoconductivity (TPC). Third, LR has a peak value at a temperature below $T_{P}$. These features are qualitatively similar to those reported in hole-doped $\mathrm{La}_{0.67} \mathrm{Ca}_{0.33}$ $\mathrm{MnO}_{3}$ (Ref. 15) and electron-doped $\mathrm{La}_{0.8} \mathrm{Te}_{0.2} \mathrm{MnO}_{3}{ }^{16}$ implying common properties in many doped manganites.

The PSMO/STO films show dramatic changes after being processed with a large current of $18 \mathrm{~mA}\left(\sim 2 \times 10^{5} \mathrm{~A} / \mathrm{cm}^{2}\right)$ at room temperature for $30 \mathrm{~min}$. As can be seen from Fig. 3(a), for the current-induced state, with decreasing temperature there is an upturn of resistance below $T_{P}$ and the resistance is greatly increased at low temperatures. Moreover, the low temperature current-voltage characteristic of this new state is nonlinear. This means a substantial electroresistance (ER) effect, as displayed in the inset. The great increase of resistance and nonlinearity are not possible to be interfacial effects since two lead $R-T$ curves measured between electrodes 1 and 2 [or between electrodes 3 and 4, see Fig. 1(inset)] prior to and after current processing are essentially the same.

Figure 3(b) presents the LR for the current-induced state from room temperature down to $\sim 80 \mathrm{~K}$. Like that in the as-prepared film, LR in this temperature range is transient. It is positive (negative) in the metallic (insulating) region. Intriguing properties appeared at lower temperatures, i.e., $30 \mathrm{~K}$. As shown in Fig. 4(a) (inset), the resistance decreased upon the irradiation of light and did not increase back to the initial value after the

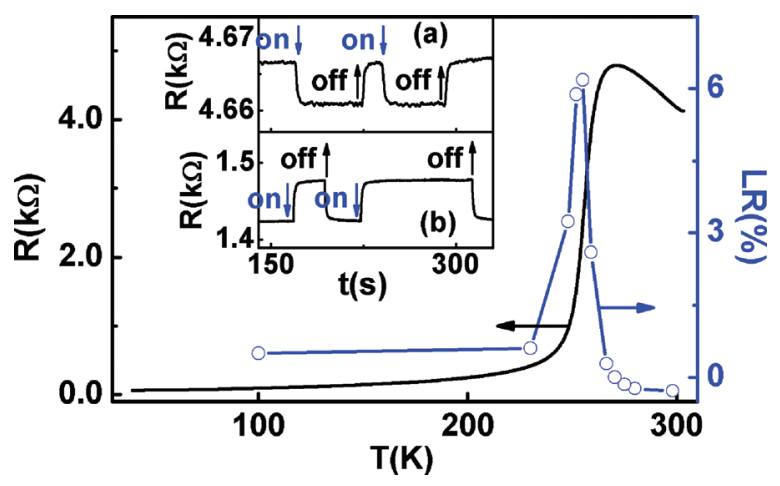

FIG. 2. (Color online) Temperature dependence of $R$ and LR for an asprepared PSMO film on STO. (Insets) Resistance vs illumination time at 283 $\mathrm{K}$ (a) and $255 \mathrm{~K}(\mathrm{~b})$.
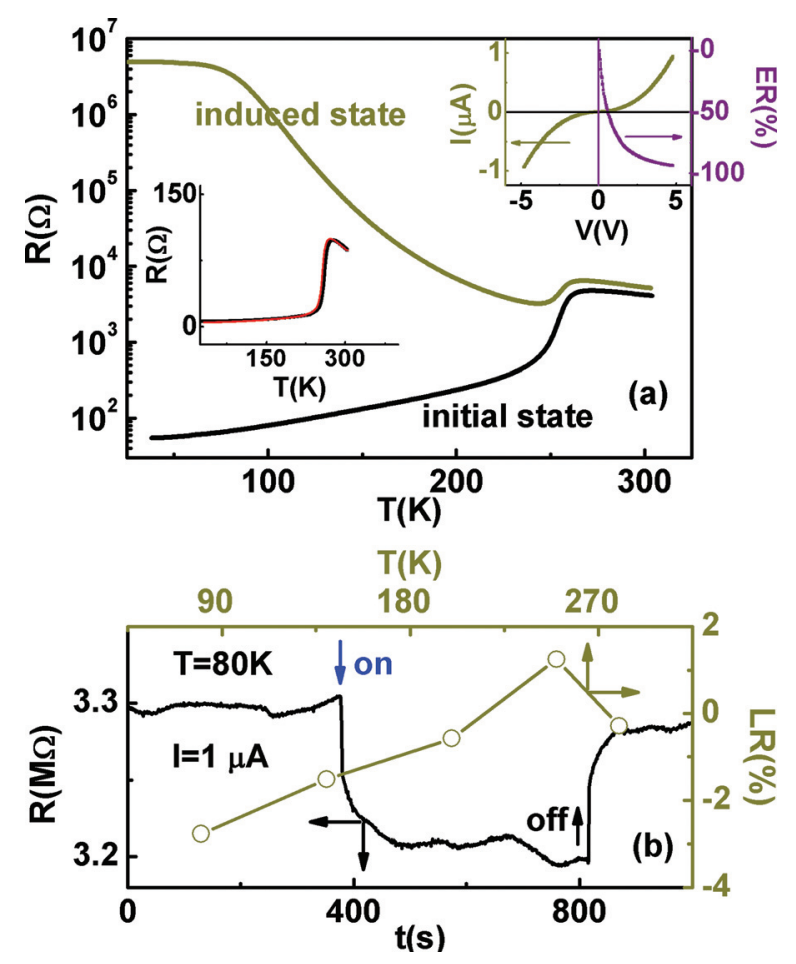

FIG. 3. (Color online) (a) $R-T$ curves $(I=1 \mu \mathrm{A})$ prior to and after current processing for PSMO on STO: (left inset) two lead $R(T)$ curves (between electrodes 1 and 2) prior to and after current processing and (right inset) $I-V$ and ER- $V$ curves for the current-induced state $(T=30 \mathrm{~K})$. (b) Temperature dependence of $\mathrm{LR}(80 \mathrm{~K} \leq T \leq 300 \mathrm{~K})$ and resistance vs illumination time for the current-induced state ( $T=80 \mathrm{~K}$, PSMO/STO).

light was turned off. This characterizes the PPC effect. If the temperature was not raised, the low resistance state could stay for hours and did not show an observable response when the light was switched on again. On the other hand, PPC could be quenched on thermal cycling. If the sample was heated to room temperature and then cooled in dark, the resistance restored to the value before illumination and PPC could be generated again. To further understand this current-induced PPC, $I-V$ curves were measured prior to, under and after light illumination [Fig. 4(a)]. Whereas distinct differences can be found for $I-V$ curves prior to and during light illumination, there is almost no change upon the stop of light. With the definition $\mathrm{PPC}=[R(\mathrm{PL})-R(\mathrm{AL})] / R(\mathrm{PL})$, where $R(\mathrm{AL})$ and $R(\mathrm{PL})$ represent the resistance after and prior to light illumination, the calculated bias-dependent PPC is displayed in Fig. 4(a) (bottom inset). With the increase of bias voltage, PPC rises from $\sim 1 \%$ at $V_{\text {bias }} \sim 0 \mathrm{~V}$ to $\sim 5 \%$ at $V_{\text {bias }}=3 \mathrm{~V}$, and keeps almost constant with further increasing of bias voltage.

Similar phenomena were also found in films grown on $\mathrm{LaAlO}_{3}$. Figure 4(b) shows the time dependence of resistance for the current-induced state with light on and off. Like that in PSMO/STO, the resistance drops quickly at the beginning of light illumination and did not recover upon the removing of light.

Such a PPC in mangnaites has not been well understood yet. Cauro et al. reported PPC in oxygen deficient $\mathrm{La}_{2 / 3} \mathrm{Sr}_{1 / 3}$ $\mathrm{MnO}_{3}$ and suggested that PPC is associated with oxygen deficiency. ${ }^{13}$ On the other hand, in samples without obvious oxygen deficiency, such as $\mathrm{Pr}_{0.7} \mathrm{Ca}_{0.3} \mathrm{MnO}_{3}$ (Ref. 9) and $\left(\mathrm{La}_{0.3} \mathrm{Nd}_{0.7}\right)_{2 / 3} \mathrm{Ca}_{1 / 3} \mathrm{MnO}_{3},{ }^{14}$ PPC was also observed. This 

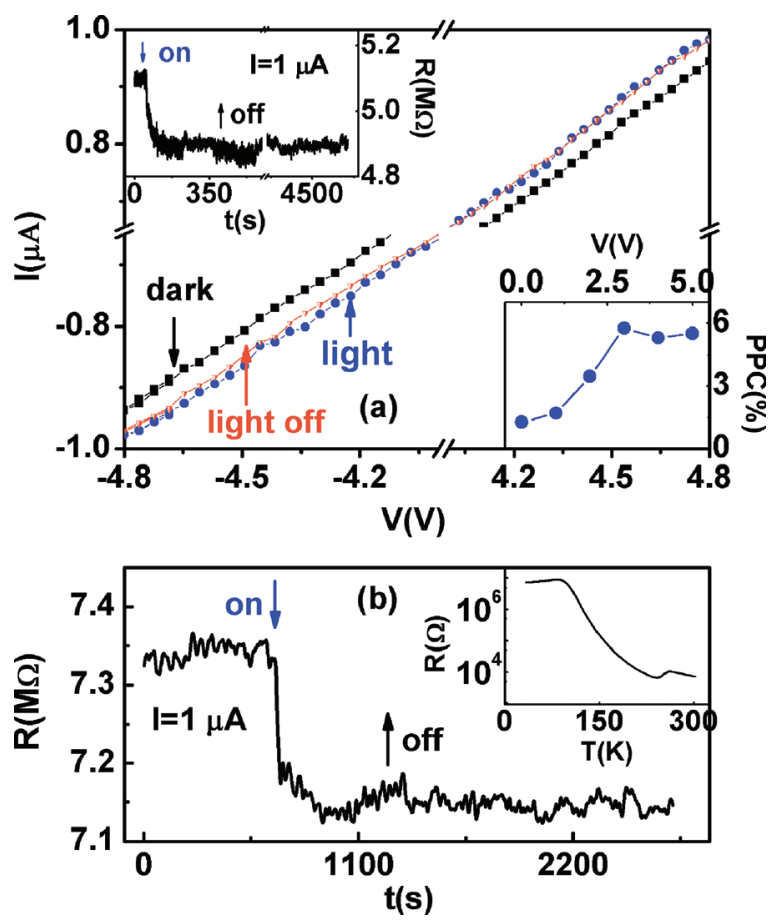

FIG. 4. (Color online) (a) $I-V$ curves prior to, under, and after light illumination for the current-induced state $(T=30 \mathrm{~K}, \mathrm{PSMO} / \mathrm{STO})$. (Insets) Resistance vs illumination time (upper) and the bias dependence of PPC (bottom). (b) PPC for the current-induced state of PSMO on LAO ( $T=30 \mathrm{~K})$. (Inset) The $R-T$ curve after current processing.

implies oxygen deficiency may not be a necessary ingredient for PPC. Alternatively, large inhomogeneity appears to be an important feature for visible light-induced PPC. ${ }^{13,14}$ Introduction of appropriate oxygen vacancies to $\mathrm{La}_{2 / 3} \mathrm{Sr}_{1 / 3} \mathrm{MnO}_{3}$ caused local lattice distortions and thus electronic imbalance. Partial substitution of $\mathrm{Ca}$ with $\mathrm{Nd}$ in $\mathrm{La}_{2 / 3} \mathrm{Ca}_{1 / 3} \mathrm{MnO}_{3}$ lead A-site disordered and could enhance the inhomogeneity. It is known that manganites are electronically soft. ${ }^{4}$ A large electric field could change the intricate balance between different phases and drive the system transiting from one state to another. It seems that the initial state at low temperature was comparably homogenous and dominated by conducting phases. With the application of the large electric field/current, the insulating portion was increased and the inhomogeneity was enhanced. As a consequence, visible light-induced PPC appeared. A full understanding of this current-induced PPC needs further study.

\section{CONCLUSIONS}

The effects of current processing on photoconductivity have been studied in $\mathrm{Pr}_{0.7} \mathrm{Sr}_{0.3} \mathrm{MnO}_{3}$ films grown on $\mathrm{SrTiO}_{3}$ and $\mathrm{LaAlO}_{3}$. The as-grown films show transient photoconductivity, which is negative above $T_{P}$ and positive below $T_{P}$. In contrast, the photoconductivity of the current-induced state is transient at high temperatures and persistent at low temperatures. Such a current-induced PPC might be related to enhanced inhomogeneity.

\section{ACKNOWLEDGMENTS}

This work has been supported by a grant of the Research Grant Council of Hong Kong (Project No. HKU 702407P) and the CRCG of the University of Hong Kong.

${ }^{1}$ R. von Helmolt, J. Wecker, B. Holzapfel, L. Schultz, and K. Samwer, Phys. Rev. Lett. 71, 2331 (1993).

${ }^{2}$ S. Jin, T. H. Tiefel, M. McCormack, R. A. Fastnacht, R. Ramesh, and L. H. Chen, Science 264, 413 (1994).

${ }^{3}$ M. B. Salamon and M. Jaime, Rev. Mod. Phys. 73, 583 (2001).

${ }^{4}$ E. Dagotto, Science 309, 257 (2005).

${ }^{5}$ Y. Tokura, Rep. Prog. Phys. 69, 797 (2006).

${ }^{6}$ A. Asamitsu,Y. Tomioka, H. Kuwahara, and Y. Tokura, Nature 388, 50 (1997).

${ }^{7}$ J. Gao and F. X. Hu, Appl. Phys. Lett. 86, 092504 (2005).

${ }^{8}$ F. X. Hu and J. Gao, Appl. Phys. Lett. 87, 152504 (2005).

${ }^{9}$ V. Kiryukhin, D. Casa, J. P. Hill, B. Keimer, A. Vigliante, Y. Tomioka, and Y. Tokura, Nature 386, 813 (1997).

${ }^{10}$ K. Miyano, T. Tanaka, Y. Tomioka, and Y. Tokura, Phys. Rev. Lett. 78, 4257 (1997)

${ }^{11}$ M. Baran, S. L. Gnatchenko, O. Y. Gorbenko, A. R. Kaul, R. Szymczak, and H. Szymczak, Phys. Rev. B 60, 9244 (1999).

${ }^{12}$ D. Talbayev, H. Zhao, G. Lüpke, A. Venimadhav, and Q. Li, Phys. Rev. B 73, 014417 (2006).

${ }^{13}$ R. Cauro, A. Gilabert, J. P. Contour, R. Lyonnet, M. G. Medici, J. C. Grenet, C. Leighton, and I. K. Schuller, Phys. Rev. B 63, 174423 (2001).

${ }^{14}$ J. M. Dai, W. H. Song, S. G. Wang, S. L. Ye, K. Y. Wang, J. J. Du, and Y. P. Sun, J. Appl. Phys. 90, 3118 (2001).

${ }^{15}$ K. Jin, C. Chen, and S. Zhao, J. Mater Sci. 42, 9617 (2007).

${ }^{16}$ G. M. Gao, C. L. Chen, L. A. Han, and X. S. Cao, J. Appl. Phys. 105, 033707 (2009). 